\title{
Varieties with ample cotangent bundle
}

\author{
Olivier Debarre
}

\begin{abstract}
The aim of this article is to provide methods for constructing smooth projective complex varieties with ample cotangent bundle. We prove that the intersection of at least $n / 2$ sufficiently ample general hypersurfaces in a complex abelian variety of dimension $n$ has ample cotangent bundle. We also discuss analogous questions for complete intersections in the projective space. Finally, we present an unpublished result of Bogomolov which states that a general linear section of small dimension of a product of sufficiently many smooth projective varieties with big cotangent bundle has ample cotangent bundle.
\end{abstract}

\section{Introduction}

Projective algebraic varieties $X$ with ample cotangent bundle have many properties: the subvarieties of $X$ are all of general type; there are finitely many nonconstant rational maps from any fixed projective variety to $X$ (see [NS82]); if $X$ is defined over $\mathbb{C}$, any entire holomorphic mapping $\mathbb{C} \rightarrow X$ is constant [Dem97, (3.1)]; if $X$ is defined over a number field $K$, the set of $K$-rational points of $X$ is conjectured to be finite [Mor95].

Although these varieties are expected to be reasonably abundant, few concrete constructions are available. The main result of this article, proved in $\S 2$, is that the intersection of at least $n / 2$ sufficiently ample general hypersurfaces in an abelian variety of dimension $n$ has ample cotangent bundle. This answers positively a question of Lazarsfeld. As a corollary, we obtain results about cohomology groups of sheaves of symmetric tensors on smooth subvarieties of abelian varieties.

In $\S 3$, mostly conjectural, we discuss analogous questions for complete intersections in the projective space.

Finally, in $\S 4$ we present an unpublished result of Bogomolov which states that a general linear section of small dimension of a product of sufficiently many smooth projective varieties with big cotangent bundle has ample cotangent bundle. This shows, in particular, that the fundamental group of a smooth projective variety with ample cotangent bundle can be any group arising as the fundamental group of a smooth projective variety.

We work over the complex numbers. Given a vector bundle $\mathcal{E}$, the projective bundle $\mathbb{P}(\mathcal{E})$ is the space of one-dimensional quotients of the fibers of $\mathcal{E}$. It is endowed with a line bundle $\mathcal{O}_{\mathbb{P}(\mathcal{E})}(1)$. We say that $\mathcal{E}$ is ample (respectively nef, respectively big) if the line bundle $\mathcal{O}_{\mathbb{P}(\mathcal{E})}(1)$ has the same property. Following [Som78], we say more generally that given an integer $k$, the vector bundle $\mathcal{E}$ is $k$-ample if, for some $m>0$, the line bundle $\mathcal{O}_{\mathbb{P}(\mathcal{E})}(m)$ is generated by its global sections and each fiber of the associated map $\mathbb{P}(\mathcal{E}) \rightarrow \mathbb{P}^{N}$ has dimension $\leqslant k$. Ampleness coincides with 0-ampleness.

Received 18 May 2004, accepted in final form 22 October 2004.

2000 Mathematics Subject Classification 14K12 (primary), 14M10, $14 \mathrm{~F} 10$ (secondary).

Keywords: ample cotangent bundle, complete intersections.

This journal is (c) Foundation Compositio Mathematica 2005. 


\section{O. DEBARRE}

\section{Subvarieties of abelian varieties}

We study the positivity properties of the cotangent bundle of a smooth subvariety of an abelian variety $A$.

\subsection{Preliminary material}

Using a translation, we identify the tangent space $T_{A, x}$ at a point $x$ of $A$ with the tangent space $T_{A, 0}$ at the origin. We begin with a classical result.

Proposition 1. Let $X$ be a smooth subvariety of an abelian variety $A$. The following properties are equivalent:

(i) the cotangent bundle $\Omega_{X}$ is $k$-ample;

(ii) for any nonzero vector $\xi$ in $T_{A, 0}$, the set $\left\{x \in X \mid \xi \in T_{X, x}\right\}$ has dimension $\leqslant k$.

Proof. The natural surjection $\left.\left(\Omega_{A}\right)\right|_{X} \rightarrow \Omega_{X}$ induces a diagram

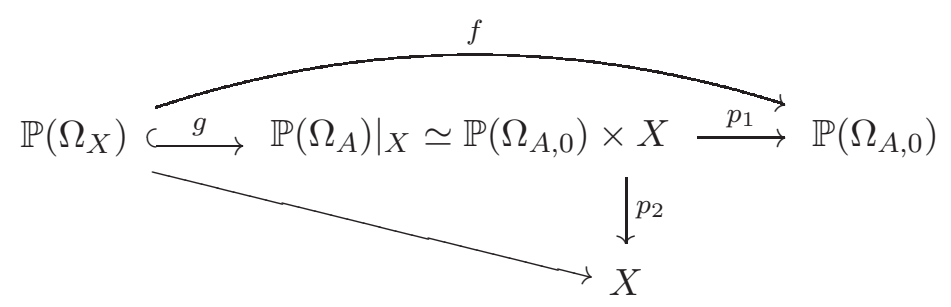

and

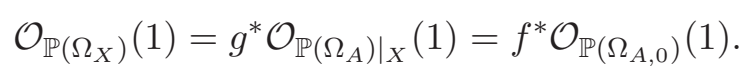

It follows that $\Omega_{X}$ is $k$-ample if and only if each fiber of $f$ has dimension $\leqslant k$ (see [Som78, Corollary 1.9]). The proposition follows, since the restriction of the projection $\mathbb{P}\left(\Omega_{X}\right) \rightarrow X$ to any fiber of $f$ is injective.

\section{Remarks 2 .}

(1) Let $d=\operatorname{dim}(X)$ and $n=\operatorname{dim}(A)$. Since $\operatorname{dim}\left(\mathbb{P}\left(\Omega_{X}\right)\right)=2 d-1$, the proof of the proposition shows that the cotangent bundle of $X$ is $(2 d-n)$-ample at best. It is always $d$-ample, and is $(d-1)$-ample except if $X$ has a nonzero vector field, which happens if and only if $X$ is stable by translation by a nonzero abelian subvariety (generated by the vector field).

(2) Many things can prevent the cotangent bundle of $X$ from being ample. Below are two examples.

(i) Assume $X \supset X_{1}+X_{2}$, where $X_{1}$ and $X_{2}$ are subvarieties of $A$ of positive dimension. For all $x_{1}$ smooth on $X_{1}$ and all $x_{2} \in X_{2}$, one has $T_{X_{1}, x_{1}} \subset T_{X, x_{1}+x_{2}}$, hence the cotangent bundle of $X$ is not $\left(\operatorname{dim}\left(X_{2}\right)-1\right)$-ample. In the Jacobian of a smooth curve $C$, the cotangent bundle of any smooth $W_{d}(C)$ is therefore exactly $(d-1)$-ample (although its normal bundle is ample).

(ii) If $A$ is (isogenous to) a product $A_{1} \times A_{2}$ and $X_{a_{2}}=X \cap\left(A_{1} \times\left\{a_{2}\right\}\right)$, the cotangent bundle of $X$ is at most $\left(2 \operatorname{dim}\left(X_{a_{2}}\right)-\operatorname{dim}\left(A_{1}\right)\right)$-ample, because of the commutative diagram

$$
\begin{array}{ccc}
\left.\mathbb{P}\left(\Omega_{X}\right)\right|_{\left(X_{a_{2}}\right)_{\mathrm{reg}}} & \stackrel{f}{\longrightarrow} & \mathbb{P}\left(\Omega_{A, 0}\right) \\
\cup & & \cup \\
\mathbb{P}\left(\Omega_{\left.\left(X_{a_{2}}\right)_{\mathrm{reg}}\right)}\right) & \longrightarrow & \mathbb{P}\left(\Omega_{A_{1}, 0}\right)
\end{array}
$$

In particular, if $\operatorname{dim}\left(X_{a_{2}}\right)>\frac{1}{2} \operatorname{dim}\left(A_{1}\right)$ for some $a_{2}$, the cotangent bundle of $X$ cannot be ample. 


\section{VARIETIES WITH AMPLE COTANGENT BUNDLE}

We will encounter the following situation twice: assume $\mathcal{F}$ and $\mathcal{G}$ are vector bundles on a projective variety $X$ that fit into an exact sequence

$$
0 \rightarrow \mathcal{F} \rightarrow V \otimes \mathcal{O}_{X} \rightarrow \mathcal{G} \rightarrow 0
$$

where $V$ is a vector space.

Lemma 3. In the situation above, if moreover $\operatorname{rank}(\mathcal{F}) \geqslant \operatorname{dim}(X)$, we have

$$
\mathcal{F}^{*} \text { ample } \Rightarrow \mathcal{G} \text { nef and big. }
$$

Proof. As in the proof of Proposition $1, \mathcal{G}$ is nef and big if and only if the morphism $f: \mathbb{P}(\mathcal{G}) \rightarrow \mathbb{P}(V)$ induced by $(2)$, which satisfies $\mathcal{O}_{\mathbb{P}(\mathcal{G})}(1)=f^{*} \mathcal{O}_{\mathbb{P}(V)}(1)$, is generically finite.

Let $d$ be the dimension of $X$, let $r$ be the dimension of $V$, let $s$ be the rank of $\mathcal{G}$, and let $G$ be the Grassmannian of vector subspaces of $V^{*}$ of dimension $s$, with tautological quotient bundle $\mathcal{Q}$ of rank $r-s \geqslant d$. The dual of the exact sequence (2) induces a map $\gamma: X \rightarrow G$ such that $\gamma^{*} \mathcal{Q}=\mathcal{F}^{*}$.

Assume that $f(\mathbb{P}(\mathcal{G}))$ has dimension $<\operatorname{dim}(\mathbb{P}(\mathcal{G}))=d+s-1$. There exists a linear subspace $W^{*}$ of $V^{*}$ of dimension $r-d-s+1$ such that $\mathbb{P}(W)$ does not meet $f(\mathbb{P}(\mathcal{G}))$. In other words, the variety $\gamma(X)$ does not meet the special Schubert variety $\left\{\Lambda \in G \mid \Lambda \cap W^{*} \neq\{0\}\right\}$, whose class is $c_{d}(\mathcal{Q})$. We obtain $\gamma(X) \cdot c_{d}(\mathcal{Q})=0$, hence $0=c_{d}\left(\gamma^{*} \mathcal{Q}\right)=c_{d}\left(\mathcal{F}^{*}\right)$, and $\mathcal{F}^{*}$ cannot be ample by [BG71, Corollary 1.2].

\subsection{Nef and big cotangent bundle}

A characterization of subvarieties of an abelian variety whose cotangent bundle is nef and big follows easily from a result of [Deb95].

Proposition 4. The cotangent bundle of a smooth subvariety $X$ of an abelian variety is nef and big if and only if $\operatorname{dim}(X-X)=2 \operatorname{dim}(X)$.

Proof. The cotangent bundle of $X$ is nef and big if and only if the morphism $f$ in (1) is generically finite onto its image $\bigcup_{x \in X} \mathbb{P}\left(\Omega_{X, x}\right)$, i.e. if the latter has dimension $2 \operatorname{dim}(X)-1$. The proposition follows from [Deb95, Theorem 2.1].

The condition $\operatorname{dim}(X-X)=2 \operatorname{dim}(X)$ implies, of course, that $2 \operatorname{dim}(X) \leqslant \operatorname{dim}(A)$. The converse holds if $X$ is nondegenerate [Deb95, Proposition 1.4]: this means that for any quotient abelian variety $\pi: A \rightarrow B$, one has either $\pi(X)=B$ or $\operatorname{dim}(\pi(X))=\operatorname{dim}(X)$. This property holds, for example, for any subvariety of a simple abelian variety. ${ }^{1}$ It has also an interpretation in terms of positivity of the normal bundle of $X$.

Proposition 5. The normal bundle of a smooth subvariety $X$ of an abelian variety is nef and big if and only if $X$ is nondegenerate.

Proof. The normal bundle $N_{X / A}$ to $X$ in $A$ is nef and big if and only if the map $f^{\prime}$ in the diagram

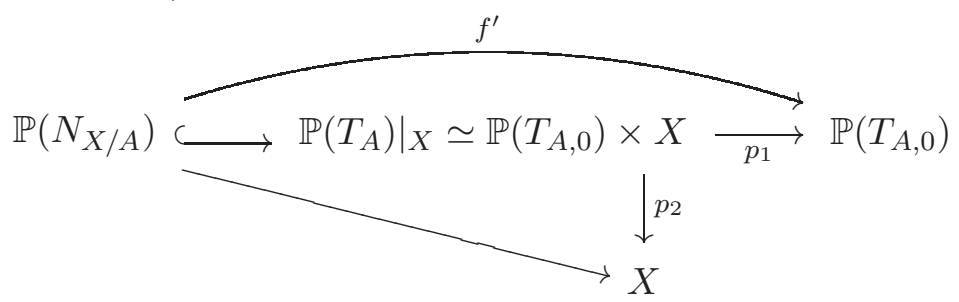

is generically finite onto its image (i.e. surjective).

\footnotetext{
${ }^{1}$ An abelian variety $A$ is simple if the only abelian subvarieties of $A$ are 0 and $A$. For more about nondegenerate subvarieties, see [Deb99, ch. VIII].
} 


\section{O. DEBARRE}

To each point $p$ in the image of $f^{\prime}$ corresponds a hyperplane $H_{p}$ in $T_{A, 0}$ such that $T_{X, x} \subset H_{p}$ for all $x$ in the image $F_{p}$ in $X$ of the fiber. This implies $T_{F_{p}, x} \subset H_{p}$ for all $x$ in $F_{p}$, hence the tangent space at the origin of the abelian variety $K_{p}$ generated by $F_{p}$ is contained in $H_{p}$ (see [Deb99, Lemme VIII.1.2]).

Since $A$ has at most countably many abelian subvarieties, the abelian variety $K_{p}$ is independent of the very general point $p$ in the image of $f^{\prime}$. Let $\pi: A \rightarrow B$ be the corresponding quotient. The differential of $\left.\pi\right|_{X}$ is not surjective at any point of $F_{p}$ since its image is contained in the hyperplane $T \pi\left(H_{p}\right)$. By generic smoothness, $\left.\pi\right|_{X}$ is not surjective.

If $X$ is nondegenerate, $\left.\pi\right|_{X}$ is generically finite onto its image, hence $F_{p}$ is finite and $f^{\prime}$ is generically finite onto its image. It follows that $N_{X / A}$ is nef and big.

Conversely, assume that $N_{X / A}$ is nef and big. Let $\pi: A \rightarrow B$ be a quotient of $X$ such that $\pi(X) \neq B$. The tangent spaces to $X$ along a general fiber of $\left.\pi\right|_{X}$ are all contained in a fixed hyperplane. This fiber is therefore finite, hence $X$ is nondegenerate.

Proposition 6. Let $X$ be a smooth subvariety of an abelian variety $A$, of dimension at most $\frac{1}{2} \operatorname{dim}(A)$. We have

$$
\Omega_{X} \text { ample } \Rightarrow N_{X / A} \text { nef and big } \Rightarrow \Omega_{X} \text { nef and big. }
$$

Proof. The first implication follows from Lemma 3 applied to the exact sequence $\left.0 \rightarrow T_{X} \rightarrow T_{A}\right|_{X} \rightarrow$ $N_{X / A} \rightarrow 0$.

The second implication follows from Propositions 4 and 5 and the fact that for a nondegenerate subvariety $X$ of $A$, the equality $\operatorname{dim}(X-X)=\min (2 \operatorname{dim}(X), \operatorname{dim}(A))$ holds [Deb95, Proposition 1.4].

\subsection{Ample cotangent bundle}

In this section, we prove that the intersection of sufficiently ample general hypersurfaces in an abelian variety $A$ has ample cotangent bundle, provided that its dimension be at most $\frac{1}{2} \operatorname{dim}(A)$.

We begin by fixing some notation. If $A$ is a smooth variety, $\partial$ a vector field on $A$, and $L$ a line bundle on $A$, we define, for any section $s$ of $L$ with divisor $H$, a section $\partial s$ of $\left.L\right|_{H}$ by the requirement that for any open set $U$ of $A$ and any trivialization $\varphi:\left.\mathcal{O}_{U} \stackrel{\sim}{\longrightarrow} L\right|_{U}$, we have $\partial s=\left.\varphi\left(\partial\left(\varphi^{-1}(s)\right)\right)\right|_{H}$ in $U \cap H$. We denote its zero locus by $H \cap \partial H$. We have an exact sequence

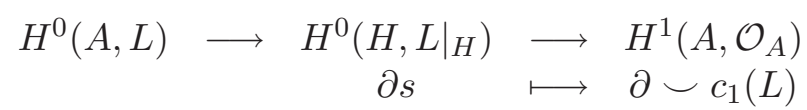

where $c_{1}(L)$ is considered as an element of $H^{1}\left(A, \Omega_{A}\right)$ and the cup product is the contraction

$$
H^{0}\left(A, T_{A}\right) \otimes H^{1}\left(A, \Omega_{A}\right) \longrightarrow H^{1}\left(A, \mathcal{O}_{A}\right)
$$

2.3.1 The simple case. We begin with the case of a simple abelian variety, where we get an explicit bound on how ample the hypersurfaces should be.

Theorem 7. Let $L_{1}, \ldots, L_{c}$ be very ample line bundles on a simple abelian variety $A$ of dimension $n$. Consider general divisors $H_{1} \in\left|L_{1}^{e_{1}}\right|, \ldots, H_{c} \in\left|L_{c}^{e_{c}}\right|$. If $e_{2}, \ldots, e_{c}$ are all $>n$, the cotangent bundle of $H_{1} \cap \cdots \cap H_{c}$ is $\max (n-2 c, 0)$-ample.

Proof. We need to prove that the fibers of the map $f$ in (1) have dimension at most $m=\max (n-$ $2 c, 0)$. This means that for $H_{i}$ general in $\left|L_{i}^{e_{i}}\right|$ and any nonzero constant vector field $\partial$ on $A$, the dimension of the set of points $x$ in $X=H_{1} \cap \cdots \cap H_{c}$ such that $\partial(x) \in T_{X, x}$ is at most $m$; in other words, that

$$
\operatorname{dim}\left(H_{1} \cap \partial H_{1} \cap \cdots \cap H_{c} \cap \partial H_{c}\right) \leqslant m
$$




\section{VARIETIES WITH AMPLE COTANGENT BUNDLE}

It is enough to treat the case $c \leqslant n / 2$. We proceed by induction on $c$, and assume that the variety $Y_{\partial}=H_{1} \cap \partial H_{1} \cap \cdots \cap H_{c-1} \cap \partial H_{c-1}$ has codimension $2 c-2$ in $A$ for all nonzero $\partial$. Let $Y_{\partial, 1}, \ldots, Y_{\partial, q}$ be its irreducible components.

Let $\mathcal{U}_{e}\left(Y_{\partial, i}\right)$ be the open set of divisors $H$ in $\left|L_{c}^{e}\right|$ such that $\left(Y_{\partial, i}\right)_{\text {red }} \cap H$ is integral of codimension 1 in $Y_{\partial, i}$. If $H \in \mathcal{U}_{e}\left(Y_{\partial, i}\right)$, we claim that $Y_{\partial, i} \cap H \cap \partial H$ has codimension 2 in $Y_{\partial, i}$. Indeed, let $s \in$ $H^{0}\left(A, L_{c}^{e}\right)$ define $H$ and set $Y=\left(Y_{\partial, i}\right)_{\text {red }}$. The scheme $Y \cap H \cap \partial H$ is the zero set in $Y \cap H$ of the section $\partial s$ defined above. In the commutative diagram,

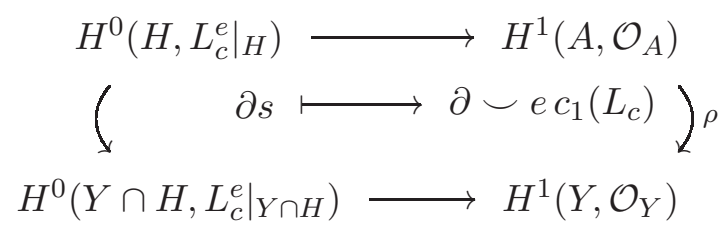

the restriction $\rho$ is injective because $Y$ generates $A$, hence $\partial s$ does not vanish identically on the integral scheme $Y \cap H$.

It follows that for $H \in \mathcal{U}_{e}\left(Y_{\partial}\right)=\bigcap_{i=1}^{q} \mathcal{U}_{e}\left(Y_{\partial, i}\right)$, the scheme $Y_{\partial} \cap H \cap \partial H$ has codimension $2 c$ in $A$. Thus, for $H_{c} \in \bigcap_{[\partial] \in \mathbb{P}\left(\Omega_{A, 0}\right)} \mathcal{U}_{e}\left(Y_{\partial}\right)$, the intersection

$$
H_{1} \cap \partial H_{1} \cap \cdots \cap H_{c} \cap \partial H_{c}
$$

has codimension $2 c$ in $A$ for all nonzero constant vector field $\partial$ on $A$ (note that when $c=1$, there is no condition on $H_{1}$ ). Lemma 12 , to be proved in $\S 2.3 .4$, shows that the complement of $\mathcal{U}_{e}\left(Y_{\partial}\right)$ in $\left|L_{c}^{e}\right|$ has codimension at least $e-1$. For $e>n$, the intersection $\bigcap_{[\partial] \in \mathbb{P}\left(\Omega_{A, 0}\right)} \mathcal{U}_{e}\left(Y_{\partial}\right)$ is therefore not empty and the theorem follows.

2.3.2 The general case. A variant of the same proof works for any abelian variety, but we lose control of the explicit lower bounds on $e_{2}, \ldots, e_{c}$.

Theorem 8. Let $L_{1}, \ldots, L_{c}$ be very ample line bundles on an abelian variety $A$ of dimension $n$. For $e_{2}, \ldots, e_{c}$ large and divisible enough positive integers and general divisors $H_{1} \in\left|L_{1}^{e_{1}}\right|, \ldots$, $H_{c} \in\left|L_{c}^{e_{c}}\right|$, the cotangent bundle of $H_{1} \cap \cdots \cap H_{c}$ is $\max (n-2 c, 0)$-ample.

Let us be more precise about the condition on the $e_{i}$. What we mean is that there exists for each $i \in\{1, \ldots, c-1\}$, a function $\delta_{i}: \mathbb{N}^{i} \rightarrow \mathbb{N}^{*}$ such that the conclusion of the theorem holds if

$$
e_{2}=e_{2}^{\prime} \delta_{1}\left(e_{1}\right), e_{3}=e_{3}^{\prime} \delta_{2}\left(e_{1}, e_{2}\right), \ldots, e_{c}=e_{c}^{\prime} \delta_{c-1}\left(e_{1}, \ldots, e_{c-1}\right) \quad \text { with } e_{2}^{\prime}, \ldots, e_{c}^{\prime}>n .
$$

Proof. We keep the setting and notation of the proof of Theorem 7. Everything goes through except when, in diagram $(4), \rho\left(\partial \smile c_{1}\left(L_{c}\right)\right)=0$. In this case, let $A^{\prime \prime}$ be the abelian subvariety of $A$ generated by $Y$ and let $A^{\prime}$ be its complement with respect to $L_{c}$, so that the addition

$$
\pi: A^{\prime} \times A^{\prime \prime} \rightarrow A
$$

is an isogeny and $\left.\left.\pi^{*} L_{c} \simeq L_{c}\right|_{A^{\prime}} \otimes L_{c}\right|_{A^{\prime \prime}}$. We have $Y=a^{\prime}+Y^{\prime \prime}$, with $a^{\prime} \in A^{\prime}, Y^{\prime \prime} \subset A^{\prime \prime}$, and $\partial \in H^{0}\left(A^{\prime}, T_{A^{\prime}}\right)$. In particular, we have an injection

$$
H^{0}\left(A, L_{c}\right) \stackrel{\pi^{*}}{\hookrightarrow} H^{0}\left(A^{\prime},\left.L_{c}\right|_{A^{\prime}}\right) \otimes H^{0}\left(A^{\prime \prime},\left.L_{c}\right|_{A^{\prime \prime}}\right) .
$$

It is, however, difficult to identify in a manner useful for our purposes the sections of $L_{c}$ inside this tensor product. Instead, we use a trick that will unfortunately force us to lose any control of the numbers involved. 


\section{O. Debarre}

The trick goes as follows. The kernel of $\pi$, being finite, is contained in the group of $r$-torsion points of $A^{\prime} \times A^{\prime \prime}$ for some positive integer $r$. Multiplication by $r$ factors as

$$
A^{\prime} \times A^{\prime \prime} \stackrel{\pi}{\longrightarrow} A \stackrel{\pi^{\prime}}{\longrightarrow} A^{\prime} \times A^{\prime \prime}
$$

and $\pi^{\prime *}\left(\left.\left.L_{c}\right|_{A^{\prime}} \otimes L_{c}\right|_{A^{\prime \prime}}\right)$ is some power $L_{c}^{e_{0}}$ of $L_{c}$. Sections of $L_{c}^{e_{0}}$ that come from $H^{0}\left(A^{\prime},\left.L_{c}\right|_{A^{\prime}}\right) \otimes$ $H^{0}\left(A^{\prime \prime},\left.L_{c}\right|_{A^{\prime \prime}}\right)$ induce a morphism from $A$ to some projective space that factors through $\pi^{\prime}$ and embeds $A^{\prime} \times A^{\prime \prime}$.

We will consider sections of $L^{e e_{0}}$ of the type $\pi^{\prime *} s$, with $s \in H^{0}\left(A^{\prime},\left.L_{c}^{e}\right|_{A^{\prime}}\right) \otimes H^{0}\left(A^{\prime \prime},\left.L_{c}^{e}\right|_{A^{\prime \prime}}\right)$. If the divisor $H$ of $s$ on $A^{\prime} \times A^{\prime \prime}$ corresponds to a degree $e$ hypersurface in $\mathcal{U}_{e}\left(\pi^{\prime}(Y)\right)$, the intersection $\pi^{\prime}(Y) \cap H$ is integral of codimension 1 in $\pi^{\prime}(Y)=\left\{r a^{\prime}\right\} \times r Y^{\prime \prime}$.

Fix a basis $\left(s_{1}^{\prime \prime}, \ldots, s_{d}^{\prime \prime}\right)$ for $H^{0}\left(A^{\prime \prime},\left.L_{c}^{e}\right|_{A^{\prime \prime}}\right)$ and write $s=\sum_{i=1}^{d} s_{i}^{\prime} \otimes s_{i}^{\prime \prime}$, so that

$$
\begin{gathered}
\pi^{\prime}(Y) \cap H=\pi^{\prime}(Y) \cap \operatorname{div}\left(\sum_{i=1}^{d} s_{i}^{\prime}\left(r a^{\prime}\right) s_{i}^{\prime \prime}\right) \\
\pi^{\prime}(Y) \cap H \cap \partial H=\pi^{\prime}(Y) \cap \operatorname{div}\left(\sum_{i=1}^{d} s_{i}^{\prime}\left(r a^{\prime}\right) s_{i}^{\prime \prime}\right) \cap \operatorname{div}\left(\sum_{i=1}^{d} \partial s_{i}^{\prime}\left(r a^{\prime}\right) s_{i}^{\prime \prime}\right) .
\end{gathered}
$$

Since $\pi^{\prime}(Y) \cap H$ is integral, $\pi^{\prime}(Y) \cap H \cap \partial H$ has codimension 2 in $\pi^{\prime}(Y)$ (hence, $Y \cap \pi^{\prime-1}(H) \cap \partial \pi^{\prime-1}(H)$ has codimension 2 in $Y)$ unless, for some complex number $\lambda$, the section $\sum_{i=1}^{d}\left(\lambda s_{i}^{\prime}+\partial s_{i}^{\prime}\right)\left(r a^{\prime}\right) s_{i}^{\prime \prime}$ of $\left.L_{c}^{e}\right|_{A^{\prime \prime}}$ vanishes on $r Y^{\prime \prime}$. In other words, if we let

$$
\Gamma_{r Y^{\prime \prime}}=\left\{\left(a_{1}, \ldots, a_{d}\right) \in \mathbb{C}^{d} \mid \sum_{i=1}^{d} a_{i} s_{i}^{\prime \prime} \text { vanishes on } r Y^{\prime \prime}\right\}
$$

and

$$
M_{\partial}=\left(\begin{array}{ccc}
s_{1}^{\prime}\left(r a^{\prime}\right) & \cdots & s_{d}^{\prime}\left(r a^{\prime}\right) \\
\partial s_{1}^{\prime}\left(r a^{\prime}\right) & \cdots & \partial s_{d}^{\prime}\left(r a^{\prime}\right)
\end{array}\right)
$$

we have $(\lambda, 1) \cdot M_{\partial} \in \Gamma_{r Y^{\prime \prime}}$. Now we may pick any collection $\left(s_{1}^{\prime}, \ldots, s_{d}^{\prime}\right)$ we like. Fix one such that the corresponding matrix $M_{\partial}$ has rank 2 for all nonzero $\partial$ and apply a square matrix $N$ of size $\operatorname{dim}\left(A^{\prime}\right)$. The condition is now that the composition

$$
\operatorname{Im}\left({ }^{\mathrm{t}} M_{\partial}\right) \subset \mathbb{C}^{d} \stackrel{{ }^{\mathrm{t}} N}{\longrightarrow} \mathbb{C}^{d} \longrightarrow \mathbb{C}^{d} / \Gamma_{r Y^{\prime \prime}}
$$

is not injective, that is either:

(i) ${ }^{\mathrm{t}} N \cdot \operatorname{Im}\left({ }^{\mathrm{t}} M_{\partial}\right) \cap \Gamma_{r Y^{\prime \prime}} \neq\{0\}$, which imposes codim $\left(\Gamma_{r Y^{\prime \prime}}\right)-1$ conditions on $N$; or

(ii) $\operatorname{Ker}\left({ }^{\mathrm{t}} N\right) \cap \operatorname{Im}\left({ }^{\mathrm{t}} M_{\partial}\right) \neq\{0\}$, which imposes $d-1$ conditions on $N$.

The 'bad' locus for $H$ corresponds to the space of matrices $N$ that satisfy either one of these properties for some nonzero $\partial \in H^{0}\left(A^{\prime}, T_{A^{\prime}}\right)$. Since, on the one hand, $d=h^{0}\left(A^{\prime \prime},\left.L_{c}^{e}\right|_{A^{\prime \prime}}\right)>e$ and, on the other hand, the codimension of $\Gamma_{r Y^{\prime \prime}}$ is the rank of the linear map $H^{0}\left(A^{\prime \prime},\left.L_{c}^{e}\right|_{A^{\prime \prime}}\right) \rightarrow$ $H^{0}\left(r Y^{\prime \prime},\left.L_{c}^{e}\right|_{r Y^{\prime \prime}}\right)$, which is greater than $e$, the codimension of the 'bad' locus is at least $e-\operatorname{dim}\left(A^{\prime}\right)+2$.

This means that for $A^{\prime \prime}$ (hence $A^{\prime}$ ) fixed, $e>n$, and $H$ general in $\left|L_{c}^{e e_{0}}\right|$, for any component $Y$ of $Y_{\partial}$ that spans (as a group) $A^{\prime \prime}$, the intersection $Y \cap H \cap \partial H$ has codimension 2 in $Y$ for all nonzero $\partial$ in $H^{0}\left(A, T_{A}\right)$.

Since $A$ has at most countably many abelian subvarieties, there are only finitely many different abelian subvarieties spanned by components of $Y_{\partial}=H_{1} \cap \partial H_{1} \cap \cdots \cap H_{c-1} \cap \partial H_{c-1}$ for $H_{1}, \ldots, H_{c-1}$ general in $\left|L_{1}^{e_{1}}\right|, \ldots,\left|L_{c-1}^{e_{c-1}}\right|$ as $\partial$ runs through the nonzero elements of $H^{0}\left(A, T_{A}\right)$. Therefore, for some positive integer $\delta$, any $e>n$, and $H$ general in $\left|L_{c}^{e \delta}\right|$, the intersection $Y_{\partial} \cap H \cap \partial H$ has 


\section{VARIETIES WITH AMPLE COTANGENT BUNDLE}

codimension 2 in $Y_{\partial}$ for all nonzero $\partial \in H^{0}\left(A, T_{A}\right)$. This proves our claim by induction on $c$ hence the theorem.

2.3.3 The four-dimensional case. In the case where the ambient abelian variety has dimension 4 , we can make the numerical conditions in Theorem 8 explicit.

Theorem 9. Let $L_{1}$ and $L_{2}$ be line bundles on an abelian fourfold $A$, with $L_{1}$ ample and $L_{2}$ very ample. For $e_{1} \geqslant 5, e_{2} \geqslant 5$, and $H_{1} \in\left|L_{1}^{e_{1}}\right|$ and $H_{2} \in\left|L_{2}^{e_{2}}\right|$ general, the surface $H_{1} \cap H_{2}$ has ample cotangent bundle.

Proof. We claim that for $H_{1}$ general in $\left|L_{1}^{e_{1}}\right|$, the scheme $Y_{\partial}=H_{1} \cap \partial H_{1}$ is an integral surface for each nonzero vector field $\partial$ on $A$. Granting the claim for the moment and using the notation of the proof of Theorem 7, the scheme $H_{1} \cap \partial H_{1} \cap H_{2}$ is then, for $H_{2} \in \mathcal{U}_{e_{2}}\left(Y_{\partial}\right)$, an integral curve that generates $A$ since its class is $e_{2} H_{1}^{2} H_{2}$. The argument of the proof of Theorem 7 applies in this case to prove that $H_{1} \cap \partial H_{1} \cap H_{2} \cap \partial H_{2}$ is finite. Taking $H_{2}$ in $\bigcap_{[\partial] \in \mathbb{P}\left(\Omega_{A, 0}\right)} \mathcal{U}_{e_{2}}\left(Y_{\partial}\right)$ (which is possible by Lemma 12 since $e_{2}>4$ ), the intersection

$$
H_{1} \cap \partial H_{1} \cap H_{2} \cap \partial H_{2}
$$

is finite for all nonzero vector fields $\partial$, which is what we need. The theorem therefore follows from the claim, proved in the next lemma.

Lemma 10. Let $A$ be an abelian variety of dimension at least 4 and let $L$ be an ample line bundle on $A$. For $e \geqslant 5$ and $H$ general in $\left|L^{e}\right|$, the scheme $H \cap \partial H$ is integral for all nonzero $\partial \in H^{0}\left(A, T_{A}\right)$.

Proof. Assume to the contrary that for some smooth $H \in\left|L^{e}\right|$, we have $H \cap \partial H=D_{1}^{\prime}+D_{2}^{\prime}$, where $D_{1}^{\prime}$ and $D_{2}^{\prime}$ are effective nonzero Cartier divisors in $H$. We follow [BD86, Proposition 1.6]: since $\operatorname{dim}(H) \geqslant 3$, there exist by the Lefschetz theorem divisors $D_{1}$ and $D_{2}$ on $A$ such that $D_{1}+D_{2} \equiv H$ and $\left.D_{i}\right|_{H} \equiv D_{i}^{\prime}$. Since $D_{i}^{\prime}$ is effective, the long exact sequence in cohomology associated with the exact sequence

$$
0 \rightarrow \mathcal{O}_{A}\left(D_{i}-H\right) \rightarrow \mathcal{O}_{A}\left(D_{i}\right) \rightarrow \mathcal{O}_{H}\left(D_{i}^{\prime}\right) \rightarrow 0
$$

shows that, for each $i \in\{1,2\}$, either $H^{0}\left(A, D_{i}\right) \neq 0$ or $H^{1}\left(A, D_{i}-H\right) \neq 0$. The case where both $H^{1}\left(A, D_{1}-H\right)$ and $H^{1}\left(A, D_{2}-H\right)$ are zero is impossible, since we would then have a section of $L^{e}$ with divisor $H \cap \partial H$ on $H$. The case where both $H^{1}\left(A, D_{1}-H\right)$ and $H^{1}\left(A, D_{2}-H\right)$ are nonzero is impossible as in [BD86] because $\operatorname{dim}(A) \geqslant 3$.

So we may assume $H^{1}\left(A, D_{2}-H\right) \neq 0$ and $H^{1}\left(A, D_{1}-H\right)=0$, and take $D_{1}$ effective such that $D_{1} \cap H=D_{1}^{\prime}$.

As in [BD86], $A$ contains an elliptic curve $E$ such that, if $B$ is the neutral component of the kernel of the composed morphism

$$
A \stackrel{\phi_{H}}{\longrightarrow} \operatorname{Pic}^{0}(A) \rightarrow \operatorname{Pic}^{0}(E),
$$

the addition map $\pi: E \times B \rightarrow A$ is an isogeny, $\partial$ is tangent to $E$, and $\pi^{*}\left(D_{1}\right)=p_{1}^{*}\left(D_{E}\right)$ for some effective divisor $D_{E}$ on $E$. Pick a basis $\left(t_{1}, \ldots, t_{d}\right)$ for $H^{0}\left(B,\left.L^{e}\right|_{B}\right)$ and a section $s$ of $L^{e}$ with divisor $H$, and write

$$
\pi^{*} s=\sum_{i=1}^{d} s_{i} \otimes t_{i}
$$

with $s_{1}, \ldots, s_{d} \in H^{0}\left(E,\left.L^{e}\right|_{E}\right)$, so that $\pi^{-1}(H \cap \partial H)$ is defined by

$$
\sum_{i=1}^{d} s_{i} \otimes t_{i}=\sum_{i=1}^{d} \partial s_{i} \otimes t_{i}=0 .
$$




\section{O. DEBARRE}

Since $D_{1}^{\prime}=H \cap D_{1}$ is contained in $H \cap \partial H$, for every point $x$ of the support of $D_{E}$, we have

$$
\operatorname{div}\left(\sum_{i=1}^{d} s_{i}(x) t_{i}\right) \subset \operatorname{div}\left(\sum_{i=1}^{d} \partial s_{i}(x) t_{i}\right) \subset B .
$$

Since these two divisors belong to the same linear series $\left|L^{e}\right|_{B} \mid$ on $B$, they must be equal and

$$
\operatorname{rank}\left(\begin{array}{ccc}
s_{1}(x) & \cdots & s_{d}(x) \\
\partial s_{1}(x) & \cdots & \partial s_{d}(x)
\end{array}\right) \leqslant 1 .
$$

Since $H$ is irreducible, the sections $s_{1}, \ldots, s_{d}$ have no common zero and the morphism $\psi_{H}: E \rightarrow$ $\mathbb{P}^{d-1}$ that they define is ramified at $x$.

The vector subspace of $H^{0}\left(E,\left.L^{e}\right|_{E}\right)$ generated by $s_{1}, \ldots, s_{d}$ only depends on $s$, not on the choice of the basis $\left(t_{1}, \ldots, t_{d}\right)$. If $b_{1}, \ldots, b_{d}$ are general points of $B$, it is also generated by $s\left(\cdot+b_{1}\right), \ldots$, $s\left(\cdot+b_{d}\right)$ and

$$
\operatorname{rank}\left(\begin{array}{ccc}
s\left(x+b_{1}\right) & \cdots & s\left(x+b_{d}\right) \\
\partial s\left(x+b_{1}\right) & \cdots & \partial s\left(x+b_{d}\right)
\end{array}\right) \leqslant 1 .
$$

Assume now that the conclusion of the lemma fails for general $H$ (and $s$ ). The point $x$ varies with $s$, but remains constant for $s$ in a hypersurface $H_{x}$ of $H^{0}\left(X, L^{e}\right)$. If $s$ is in

$$
H_{x}^{\prime}=H_{x} \cap\left\{t \in H^{0}\left(X, L^{e}\right) \mid t\left(x+b_{1}\right)=t\left(x+b_{2}\right)=0\right\}
$$

it also satisfies $\partial s\left(x+b_{1}\right)=\partial s\left(x+b_{2}\right)=0$. Since $H_{x}^{\prime}$ has codimension at most 3 in $H^{0}\left(X, L^{e}\right)$, this means that $L^{e}$ is not 3 -jet ample and contradicts Theorem 1 of [BS97]: the lemma is proved.

Remark 11. Let $A$ be an abelian fourfold that contains no elliptic curves. The proof of Lemma 10 shows that for any smooth ample hypersurface $H$ in $A$ and any nonzero $\partial \in H^{0}\left(A, T_{A}\right)$, the scheme $H \cap \partial H$ is integral. It follows that for $L$ very ample, $e \geqslant 5$, and $H^{\prime} \in\left|L^{e}\right|$ general, the surface $H \cap H^{\prime}$ has ample cotangent bundle (compare with Theorem 7).

2.3.4 Proof of the lemma. We prove the lemma used in the proofs of all three theorems.

Lemma 12. Let $Y$ be an integral subscheme of $\mathbb{P}^{n}$ of dimension at least 2 and let $\mathcal{V}_{e, n}$ be the projective space of hypersurfaces of degree $e$ in $\mathbb{P}^{n}$. The codimension of the complement $\mathcal{V}_{e}(Y)$ of

$$
\mathcal{U}_{e}(Y)=\left\{F \in \mathcal{V}_{e, n} \mid Y \cap F \text { is integral of codimension } 1 \text { in } Y\right\}
$$

in $\mathcal{V}_{e, n}$ is at least $e-1$.

Proof. By taking hyperplane sections, we may assume that $Y$ is a surface. We proceed by induction on $n$. For $n=2$, this codimension is

$$
\min _{1 \leqslant k \leqslant e-1}\left(\left(\begin{array}{c}
e+2 \\
2
\end{array}\right)-\left(\begin{array}{c}
k+2 \\
2
\end{array}\right)-\left(\begin{array}{c}
e-k+2 \\
2
\end{array}\right)+1\right)=e-1 .
$$

Assume $n \geqslant 3$. Let $\mathcal{V}$ be a component of $\mathcal{V}_{e}(Y)$ of maximal dimension and let $\mathcal{C}_{e, p}$ be the linear subspace of $\mathcal{V}_{e, n}$ that consists of cones with vertex a point $p$. If $\mathcal{V}$ does not meet $\mathcal{C}_{e, p}$, we have

$$
\operatorname{codim}(\mathcal{V}) \geqslant \operatorname{dim}\left(\mathcal{C}_{e, p}\right)-1=\left(\begin{array}{c}
n-1+e \\
e
\end{array}\right)-1>e-1
$$

and the lemma is proved. We will therefore assume that $\mathcal{V}$ meets $\mathcal{C}_{e, p}$. Let $\pi: \mathbb{P}^{n}-\{p\} \rightarrow \mathbb{P}^{n-1}$ be a projection. If $F$ is general in $\mathcal{V} \cap \mathcal{C}_{e, p}$, either: 


\section{VARIETIES WITH AMPLE COTANGENT BUNDLE}

(i) $\pi(F) \cap \pi(Y)$ is not integral of dimension 1, the induction hypothesis yields

$$
\begin{aligned}
\operatorname{codim}_{\mathcal{V}_{e}} \mathcal{V} & \geqslant \operatorname{codim}_{\mathcal{C}_{e, p}}\left(\mathcal{V} \cap \mathcal{C}_{e, p}\right) \\
& \geqslant \operatorname{codim}_{\mathcal{V}_{e, n-1}}\left(\mathcal{V}_{e}(\pi(Y)) \cap \mathcal{V}_{e, n-1}\right) \\
& \geqslant e-1
\end{aligned}
$$

and the lemma is proved; or

(ii) the curve $\pi(F) \cap \pi(Y)$ is integral of dimension 1, but is contained in the locus $E$ over which the finite morphism $\left.\pi\right|_{Y}: Y \rightarrow \pi(Y)$ is not an isomorphism.

In case (ii), if $n \geqslant 4$, the morphism $\left.\pi\right|_{Y}$ is birational, $E$ has dimension at most 1 , hence

$$
\begin{aligned}
\operatorname{codim}_{\mathcal{V}_{e, n} \mathcal{V}} & \geqslant \operatorname{codim}_{\mathcal{C}_{e, p}}\left(\mathcal{V} \cap \mathcal{C}_{e, p}\right) \\
& \geqslant \operatorname{codim}_{\mathcal{V}_{e, n-1}}\left\{F \in \mathcal{V}_{e, n-1} \mid F \text { contains a component of } E\right\} \\
& \geqslant e+1
\end{aligned}
$$

where the last inequality holds because any $e+1$ points in $\mathbb{P}^{n-1}$ impose independent conditions on hypersurfaces of degree $e$. The lemma is proved in this case.

We are reduced to the case $n=3$ : the curve $C=\pi(F) \subset \mathbb{P}^{2}$ is integral and its inverse image $F \cap Y$ by $\left.\pi\right|_{Y}: Y \rightarrow \mathbb{P}^{2}$, is reduced but reducible.

We consider the following degeneration. Denote by $G$ an equation for $Y$; the surface $Y_{t}$ defined by $G\left(t x_{0}, x_{1}, x_{2}, x_{3}\right)=0$ is projectively equivalent to $Y$ for $t \neq 0$, whereas $Y_{0}$ is the cone with vertex $(1,0,0,0)$ and base $Y \cap\left(x_{0}=0\right)$. We may therefore assume that $Y$ is an integral cone with vertex a point $p^{\prime} \neq p$ and we let $\pi^{\prime}: Y-\left\{p^{\prime}\right\} \rightarrow C^{\prime} \subset \mathbb{P}^{2}$ be the projection. Let $O$ be the intersection of the line $p p^{\prime}$ with the plane $\mathbb{P}^{2}$. Pick a line $L$ in $\mathbb{P}^{2}$, avoiding $O$, and consider the projections $C-\{O\} \rightarrow L$ and $C^{\prime} \rightarrow L$ from $O$ (the point $O$ might be on $C$ (if $F \ni p^{\prime}$ ), but is not on $C^{\prime}$, because $\left.p \notin Y\right)$. The maps

$$
\begin{array}{ccc}
(F \cap Y)-\left\{p^{\prime}\right\} & \longrightarrow & (C-\{O\}) \times_{L} C^{\prime} \\
x & \longmapsto & \left(\pi(x), \pi^{\prime}(x)\right)
\end{array}
$$

and

$$
\begin{array}{ccc}
(C-\{O\}) \times{ }_{L} C^{\prime} & \longrightarrow & (F \cap Y)-\left\{p^{\prime}\right\} \\
\left(y, y^{\prime}\right) & \longmapsto & p y \cap p^{\prime} y^{\prime}
\end{array}
$$

are inverse to each other. Therefore, given the integral curve $C^{\prime}$, we need to study the dimension of the set of curves $C$ of degree $e$ for which the curve $C \times{ }_{L} C^{\prime}$ is reducible.

Using the same trick as above, we degenerate $C^{\prime}$ to the union $C_{0}^{\prime}$ of $\operatorname{deg}\left(C^{\prime}\right)$ distinct lines through some point. At the limit, $C \times{ }_{L} C_{0}^{\prime}$ is the union of $\operatorname{deg}\left(C^{\prime}\right)$ curves isomorphic to $C$. If $C$ is integral, the projection $C \times{ }_{L} C_{0}^{\prime} \rightarrow C_{0}^{\prime}$ has the property that every irreducible component of $C_{0}^{\prime}$ is dominated by a unique component of $C \times{ }_{L} C_{0}^{\prime}$, and the set of 'bad' curves has codimension $e-1$ as we saw in the case $n=2$. This property of the projection, begin open, carries over to $C \times{ }_{L} C^{\prime} \rightarrow C^{\prime}$. This finishes the proof of the lemma.

\subsection{Cohomology of symmetric tensors}

Let $X$ be a smooth subvariety of an abelian variety. We are interested in the cohomology groups of the symmetric powers $\mathbb{S}^{r} \Omega_{X}$.

Proposition 13. Let $A$ be an abelian variety of dimension $n$ and let $X$ be a smooth subvariety of codimension $c$ of $A$ with ample normal bundle. For $r \geqslant 0$, the restriction

$$
H^{q}\left(A, \mathbb{S}^{r} \Omega_{A}\right) \longrightarrow H^{q}\left(X, \mathbb{S}^{r} \Omega_{X}\right)
$$

is bijective for $q<n-2 c$ and injective for $q=n-2 c$. 


\section{O. DEBARRE}

Remark. For the case $q=0$, Bogomolov gave in [Bog78] a very nice proof that goes as follows. Arguing as in the proof of Proposition 4, we find that the morphism $f$ of (1) is surjective whenever $X-X=A$. Any fiber of $f$ is isomorphic to its projection to $X$, which is the zero locus of a section of $N_{A / X}$. It follows that when $N_{A / X}$ is ample and $c<n-c$, the fibers of $f$ are connected, hence $f_{*} \mathcal{O}_{\mathbb{P}\left(\Omega_{X}\right)}(r) \simeq \mathcal{O}_{\mathbb{P}\left(\Omega_{A, 0}\right)}(r)$, from which we get, for all $r \geqslant 0$,

$$
\begin{aligned}
H^{0}\left(X, \mathbb{S}^{r} \Omega_{X}\right) & \simeq H^{0}\left(\mathbb{P}\left(\Omega_{X}\right), \mathcal{O}_{\mathbb{P}\left(\Omega_{X}\right)}(r)\right) \\
& \simeq H^{0}\left(\mathbb{P}\left(\Omega_{A, 0}\right), \mathcal{O}_{\mathbb{P}\left(\Omega_{A, 0}\right)}(r)\right) \simeq H^{0}\left(A, \mathbb{S}^{r} \Omega_{A}\right) .
\end{aligned}
$$

Proof. We follow the ideas of [Sch92]. The symmetric powers of the exact sequence $0 \rightarrow N_{X / A}^{*} \rightarrow$ $\left.\Omega_{A}\right|_{X} \rightarrow \Omega_{X} \rightarrow 0$ yield, for each $r>0$, a long exact sequence

$$
\left.0 \rightarrow \wedge^{c} N_{X / A}^{*} \otimes \mathbb{S}^{r-c} \Omega_{A} \rightarrow \cdots \rightarrow N_{X / A}^{*} \otimes \mathbb{S}^{r-1} \Omega_{A} \rightarrow \mathbb{S}^{r} \Omega_{A}\right|_{X} \rightarrow \mathbb{S}^{r} \Omega_{X} \rightarrow 0 .
$$

By Le Potier's vanishing theorem [Lep75, Laz04, Remark 7.3.6], $H^{q}\left(X, \wedge^{i} N_{X / A}^{*}\right)$ vanishes for $n-$ $c-q>c-i$ and $i>0$. Since $\Omega_{A}$ is trivial, we get, by an elementary homological algebra argument [Sch92, Lemma, p. 176],

$$
H^{q}\left(X, \operatorname{Ker}\left(\left.\mathbb{S}^{r} \Omega_{A}\right|_{X} \rightarrow \mathbb{S}^{r} \Omega_{X}\right)\right)=0 \quad \text { for all } q \leqslant n-2 c .
$$

The proposition now follows from the fact that the restriction $H^{q}\left(A, \mathcal{O}_{A}\right) \rightarrow H^{q}\left(X, \mathcal{O}_{X}\right)$, hence also the restriction $H^{q}\left(A, \mathbb{S}^{r} \Omega_{A}\right) \rightarrow H^{q}\left(X,\left.\mathbb{S}^{r} \Omega_{A}\right|_{X}\right)$, is bijective for $q \leqslant n-2 c$ (see [Som79]).

Sommese proved [Som78, Proposition (1.7)] that for any $k$-ample vector bundle $\mathcal{E}$ on a projective variety $X$ and any coherent sheaf $\mathcal{F}$ on $X$,

$$
H^{q}\left(X, \mathbb{S}^{r} \mathcal{E} \otimes \mathcal{F}\right)=0
$$

for all $q>k$ and $r \gg 0$. Theorem 7 and Proposition 13 therefore imply the following.

Corollary 14. Let $X$ be the intersection of $c$ sufficiently ample general hypersurfaces (for which (5) is satisfied) in an abelian variety $A$ of dimension $n$. We have

$$
h^{q}\left(X, \mathbb{S}^{r} \Omega_{X}\right) \begin{cases}=0 & \text { for } q>\max \{n-2 c, 0\} \text { and } r \gg 0 \\ =h^{q}\left(A, \mathbb{S}^{r} \Omega_{A}\right) & \text { for } q<n-2 c \text { and } r \geqslant 0 \\ \geqslant h^{q}\left(A, \mathbb{S}^{r} \Omega_{A}\right) & \text { for } q=n-2 c \text { and } r \geqslant 0 .\end{cases}
$$

\section{Subvarieties of the projective space}

We now study the positivity properties of the cotangent bundle of a smooth subvariety of the projective space.

\subsection{Big twisted cotangent bundle}

If $X$ is a smooth subvariety of $\mathbb{P}^{n}$ of dimension $d$, we let $\gamma_{X}: X \rightarrow G\left(d, \mathbb{P}^{n}\right)$ be the Gauss map. We denote by $\mathcal{S}$ the universal subbundle and by $\mathcal{Q}$ the universal quotient bundle on $G\left(d, \mathbb{P}^{n}\right)$. We have $\gamma_{X}^{*} \mathcal{Q}=N_{X / \mathbb{P}^{n}}(-1)$ and a commutative diagram

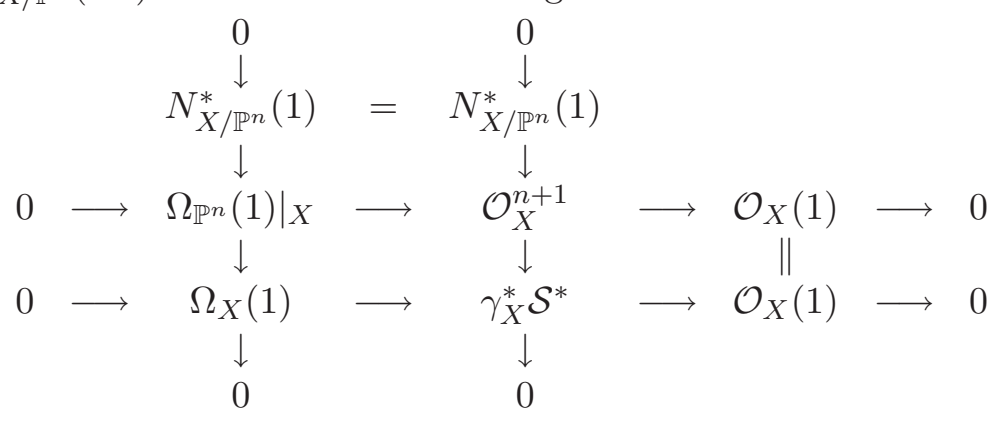

The following result is proved as Propositions 4 and 6 . 


\section{VARIETIES WITH AMPLE COTANGENT BUNDLE}

Proposition 15. Let $X$ be a smooth subvariety of dimension $d$ of $\mathbb{P}^{n}$.

(i) If $\gamma_{X}^{*} \mathcal{S}^{*}$ is big, $2 d \leqslant n$.

(ii) If $2 d \leqslant n$ and $N_{X / P^{n}}(-1)$ is ample, $\gamma_{X}^{*} \mathcal{S}^{*}$ is nef and big.

Similarly, with the same ideas, we prove an analog of Theorem 7.

Theorem 16. Let $X$ be a general complete intersection in $\mathbb{P}^{n}$ of multidegree $\left(e_{1}, \ldots, e_{c}\right)$. If $e_{1} \geqslant 2$ and $e_{2}, \ldots, e_{c}$ are all $\geqslant n+2$, the vector bundle $\gamma_{X}^{*} \mathcal{S}^{*}$ is $\max (n-2 c, 0)$-ample.

Proof. We need to prove that the fibers of the composed map

$$
\mathbb{P}\left(\gamma_{X}^{*} \mathcal{S}^{*}\right) \hookrightarrow \mathbb{P}^{n} \times X \stackrel{p_{1}}{\longrightarrow} \mathbb{P}^{n}
$$

analogous to the map $f$ in diagram (3) have dimension at most $m=\max (n-2 c, 0)$. This means that for $H_{i}$ general in $\left|\mathcal{O}_{\mathbb{P}^{n}}\left(e_{i}\right)\right|$ and for any $t$ in $\mathbb{P}^{n}$, the dimension of the set of points $x$ in $X$ such that $t \in \mathbb{T}_{X, x}$ is at most $m$. Pick coordinates and write $t=\left(t_{0}, \ldots, t_{n}\right)$. If $s$ is an equation of a hypersurface $H$, we let $\partial_{t} H$ be the hypersurface with equation $\partial_{t} s=\sum_{i=0}^{n} t_{i}\left(\partial s / \partial x_{i}\right)$. With this notation, we want

$$
\operatorname{dim}\left(H_{1} \cap \partial_{t} H_{1} \cap \cdots \cap H_{c} \cap \partial_{t} H_{c}\right) \leqslant m .
$$

As in the proof of Theorem 7 , we proceed by induction on $c$, assuming $c \leqslant n / 2$. When $c=1$, it is clear that $e_{1} \geqslant 2$ is sufficient.

Assume that $Y_{t}=H_{1} \cap \partial_{t} H_{1} \cap \cdots \cap H_{c-1} \cap \partial_{t} H_{c-1}$ has (pure) codimension $2 c-2$ in $\mathbb{P}^{n}$, with irreducible components $Y_{t, 1}, \ldots, Y_{t, m}$. Set $Y=\left(Y_{t, i}\right)_{\text {red }}$; it follows from Lemma 12 that $Y \cap H$ is integral of codimension 1 in $Y$ for $H$ outside a closed subset of codimension $\geqslant d-1$ in $\left|\mathcal{O}_{\mathbb{P}^{n}}(d)\right|$.

Assume that this is the case. If $\operatorname{codim}_{Y}\left(Y \cap H \cap \partial_{t} H\right) \leqslant 1$, the section $\partial_{t} s$ must vanish on $Y \cap H$. Since the restriction

$$
H^{0}\left(Y, \mathcal{O}_{Y}(d-1)\right) \rightarrow H^{0}\left(Y \cap H, \mathcal{O}_{Y \cap H}(d-1)\right)
$$

is injective, it must also vanish on $Y$. Since any $d$ distinct points of $Y$ impose independent conditions on elements of $\left|\mathcal{O}_{\mathbb{P} n}(d-1)\right|$ and the map $\partial_{t}: H^{0}\left(\mathbb{P}^{n}, \mathcal{O}_{\mathbb{P}}(d)\right) \rightarrow H^{0}\left(\mathbb{P}^{n}, \mathcal{O}_{\mathbb{P} n}(d-1)\right)$ is surjective, we have proved that the set of hypersurfaces $H$ in $\left|\mathcal{O}_{\mathbb{P}^{n}}(d)\right|$ such that $\operatorname{codim}_{Y_{t}}\left(Y_{t} \cap H \cap \partial_{t} H\right) \leqslant 1$ has codimension $\geqslant d-1$ in $\left|\mathcal{O}_{\mathbb{P}^{n}}(d)\right|$. The theorem follows.

Corollary 17. Let $X$ be a general complete intersection in $\mathbb{P}^{n}$ of multidegree $\left(e_{1}, \ldots, e_{c}\right)$. If $e_{1} \geqslant 2$ and $e_{2}, \ldots, e_{c}$ are all $\geqslant n+2$, and $c \geqslant n / 2$, the vector bundle $\Omega_{X}(1)$ is big.

When $X$ is a surface (i.e. $c=n-2$ ), results of Bogomolov [Bog79, Bog78] give the much better result that $\Omega_{X}\left(-\frac{1}{5} K_{X}\right)$ is big.

Proof. The last row of diagram (6) yields, for all positive integers $r$, an exact sequence

$$
0 \rightarrow \mathbb{S}^{r}\left(\Omega_{X}(1)\right) \rightarrow \mathbb{S}^{r}\left(\gamma_{X}^{*} \mathcal{S}^{*}\right) \rightarrow \mathbb{S}^{r-1}\left(\gamma_{X}^{*} \mathcal{S}^{*}\right) \otimes \mathcal{O}_{X}(1) \rightarrow 0 .
$$

It follows from Theorem 16 that for $r \gg 0$, we have

$$
H^{q}\left(X, \mathbb{S}^{r}\left(\Omega_{X}(1)\right)\right)=0 \quad \text { for } q>1 .
$$

On the other hand, if $d=n-c$, the coefficient of $r^{2 d-1} /((2 d-1) !)$ in the polynomial $\chi\left(X, \mathbb{S}^{r}\left(\Omega_{X}(1)\right)\right)$ is

$$
\begin{aligned}
s_{d}\left(\Omega_{X}(1)^{*}\right) & =\left[\prod_{i=1}^{c}\left(1+\left(e_{i}-1\right) h\right)(1-h)\right]_{d} \\
& =\sum_{1 \leqslant i_{1}<\cdots<i_{d} \leqslant c}\left(e_{i_{1}}-1\right) \cdots\left(e_{i_{d}}-1\right)-\sum_{1 \leqslant i_{1}<\cdots<i_{d-1} \leqslant c}\left(e_{i_{1}}-1\right) \cdots\left(e_{i_{d-1}}-1\right) .
\end{aligned}
$$




\section{O. DEBARRE}

Since $c \geqslant n / 2$ this is positive, so that by (7), we have, for $r \gg 0$,

$$
h^{0}\left(X, \mathbb{S}^{r}\left(\Omega_{X}(1)\right)\right) \geqslant \chi\left(X, \mathbb{S}^{r}\left(\Omega_{X}(1)\right)\right)=\alpha r^{2 d-1}+O\left(r^{2 d-2}\right)
$$

for some $\alpha>0$. This shows that $\Omega_{X}(1)$ is big.

\subsection{Conjectures}

By analogy with Theorem 7, it is tempting to conjecture the following generalization of a question formulated by Schneider in [Sch92, p. 180].

Conjecture 18. The cotangent bundle of the intersection in $\mathbb{P}^{n}$ of at least $n / 2$ general hypersurfaces of sufficiently high degrees is ample.

Ampleness can be characterized cohomologically as follows.

Proposition 19. Let $X$ be a projective variety and let $L$ be an ample line bundle on $X$. $A$ vector bundle $\mathcal{E}$ on $X$ is ample if and only if, for any integer $m$, we have $H^{q}\left(X, \mathbb{S}^{r} \mathcal{E} \otimes L^{m}\right)=0$ for all $q>0$ and $r \gg 0$.

Proof. Let $\mathcal{F}$ be an arbitrary coherent sheaf on $X$. It has a possibly nonterminating resolution

$$
\cdots \rightarrow \mathcal{E}_{2} \rightarrow \mathcal{E}_{1} \rightarrow \mathcal{E}_{0} \rightarrow \mathcal{F} \rightarrow 0
$$

by locally free sheaves that are direct sums of powers of $L$. Therefore, $H^{q}\left(X, \mathbb{S}^{r} \mathcal{E} \otimes \mathcal{E}_{j}\right)=0$ for all $j \in\{0, \ldots, \operatorname{dim}(X)\}$, all $q>0$ and $r \gg 0$, and this implies $H^{q}\left(X, \mathbb{S}^{r} \mathcal{E} \otimes \mathcal{F}\right)=0$ for all $q>0$ and $r \gg 0$. This proves that $\mathcal{E}$ is ample [Laz04, Theorem 6.1.10].

Conjecture 18 therefore has the following equivalent cohomological formulation.

Conjecture 20. Let $X$ be as in Conjecture 18. For any integer $m$, we have $H^{q}\left(X,\left(\mathbb{S}^{r} \Omega_{X}\right)(m)\right)=0$ for all $q>0$ and $r \gg 0$.

Let $X$ be a smooth projective variety of dimension $d$ with $\omega_{X}$ ample and let $L$ be a line bundle on $X$. It follows from [Dem97, Theorem 14.1], that $H^{d}\left(X, \mathbb{S}^{r} \Omega_{X} \otimes L\right)$ vanishes for $r \gg 0$. This leads us to think that the following stronger form of Conjecture 20 might be true.

Conjecture 21. Let $X$ be the intersection in $\mathbb{P}^{n}$ of $c$ general hypersurfaces of sufficiently high degrees and let $m$ be an integer. For $r \gg 0$, we have

$$
H^{q}\left(X,\left(\mathbb{S}^{r} \Omega_{X}\right)(m)\right)=0
$$

except for $q=\max \{n-2 c, 0\}$.

Remarks 22.

(1) For any smooth subvariety $X$ of $\mathbb{P}^{n}$ of codimension $c$, the vanishing (8) holds for $q<n-2 c$ and $r \geqslant m+2$ by [Sch92, Theorem 1.1], and for $q=n-c$ by Demailly's theorem. In particular, Conjecture 21 holds for $c \leqslant 1$.

(2) Under the hypotheses of Conjecture 21, one checks that the leading coefficient of the polynomial $\chi\left(\mathbb{P}\left(\Omega_{X}\right), \mathcal{O}_{\mathbb{P}\left(\Omega_{X}\right)}(r)\right)$ has sign $(-1)^{\max \{n-2 c, 0\}}$. This is compatible with the conjecture.

\section{Bogomolov's construction of varieties with ample cotangent bundle}

We present here an old unpublished construction of Bogomolov that produces varieties with ample cotangent bundle as linear sections of products of varieties with big cotangent bundle (a differentialgeometric version of this construction appeared later in [Won84]). Everything in this section is due to Bogomolov. 


\section{VARIETIES WITH AMPLE COTANGENT BUNDLE}

Proposition 23 (Bogomolov). Let $X_{1}, \ldots, X_{m}$ be smooth projective varieties with big cotangent bundle, all of dimension at least $d>0$. Let $V$ be a general linear section of $X_{1} \times \cdots \times X_{m}$. If $\operatorname{dim}(V) \leqslant(d(m+1)+1) / 2(d+1)$, the cotangent bundle of $V$ is ample.

Proof. Since $\Omega_{X_{i}}$ is big, there exist a proper closed subset $B_{i}$ of $\mathbb{P}\left(\Omega_{X_{i}}\right)$ and an integer $q$ such that for each $i$, the sections of $\mathcal{O}_{\mathbb{P}\left(\Omega_{X_{i}}\right)}(q)$, i.e. the sections of $\mathbb{S}^{q} \Omega_{X_{i}}$, define an injective morphism

$$
f_{i}: \mathbb{P}\left(\Omega_{X_{i}}\right)-B_{i} \longrightarrow \mathbb{P}^{n_{i}} .
$$

Lemma 24. Let $X$ be a smooth subvariety of $\mathbb{P}^{n}$ and let $B$ be a subvariety of $\mathbb{P}\left(\Omega_{X}\right)$. A general linear section $V$ of $X$ of dimension at most $\frac{1}{2} \operatorname{codim}(B)$ satisfies

$$
\mathbb{P}\left(\Omega_{V}\right) \cap B=\varnothing .
$$

Proof. Consider the variety

$$
\left\{((t, x), \Lambda) \in B \times G\left(n-c, \mathbb{P}^{n}\right) \mid x \in X \cap \Lambda, t \in T_{X, x} \cap T_{\Lambda, x}\right\} .
$$

The fibers of its projection to $B$ have codimension $2 c$, hence it does not dominate $G\left(n-c, \mathbb{P}^{n}\right)$ as soon as $2 c>\operatorname{dim}(B)$. This is equivalent to $2(\operatorname{dim}(X)-\operatorname{dim}(V))-1 \geqslant 2 \operatorname{dim}(X)-1-\operatorname{codim}(B)$ and the lemma is proved.

Let $B_{i}^{\prime}$ be the (conical) inverse image of $B_{i}$ in the total space of the tangent bundle of $X_{i}$. Let $V$ be a general linear section of $X_{1} \times \cdots \times X_{m}$ and set $a=m+1-2 \operatorname{dim}(V)$.

If $t=\left(t_{1}, \ldots, t_{m}\right)$, with $t_{i} \in T_{X_{i}, x_{i}}$, is a nonzero tangent vector to $V$, the lemma implies that there are at least $a$ values of the index $i$ for which $t_{i} \notin B_{i}^{\prime}$. If, say, $t_{1}$ is not in $B_{1}^{\prime}$, there exists a section of $\mathbb{S}^{q} \Omega_{X_{1}}$ that does not vanish at $t_{1}$. This section induces, via the projection $V \rightarrow X_{1}$, a section of $\mathbb{S}^{q} \Omega_{V}$ that does not vanish at $t$. It follows that $\mathcal{O}_{\mathbb{P}\left(\Omega_{V}\right)}(q)$ is base-point-free and its sections define a morphism $f: \mathbb{P}\left(\Omega_{V}\right) \longrightarrow \mathbb{P}^{n}$.

We need to show that $f$ is finite. Assume to the contrary that a curve $C$ in $\mathbb{P}\left(\Omega_{V}\right)$ through $t$ is contracted. Since the restriction of the projection $\pi: \mathbb{P}\left(\Omega_{V}\right) \rightarrow V$ to any fiber of $f$ is injective, and since $f_{i}$ is injective, the argument above proves that the curve $\pi(C)$ is contracted by each projection $p_{i}: V \rightarrow X_{i}$ such that $t_{i} \notin B_{i}^{\prime}$.

The following lemma leads to a contradiction when $2 \operatorname{dim}(V) \leqslant a d+1$. This proves the proposition.

LEmma 25. Let $V$ be a general linear section of a product $X \times Y$ in a projective space. If $2 \operatorname{dim}(V) \leqslant$ $\operatorname{dim}(X)+1$, the projection $V \rightarrow X$ is finite.

Proof. Let $\mathbb{P}^{n}$ be the ambient projective space and let $G=G\left(n-c, \mathbb{P}^{n}\right)$. Set

$$
I=\overline{\left\{\left(x, y, y^{\prime}, \Lambda\right) \in X \times Y \times Y \times G \mid y \neq y^{\prime},(x, y) \in \Lambda,\left(x, y^{\prime}\right) \in \Lambda\right\}} .
$$

General fibers of the projection $I \rightarrow X \times Y \times Y$ have codimension $2 c$. If $\Lambda$ is general in $G$ and $V=(X \times Y) \cap \Lambda$, the fiber of the projection $I \rightarrow G$ at $\Lambda$, which is isomorphic to

$$
\overline{\left\{\left((x, y),\left(x, y^{\prime}\right)\right) \in V \times V \mid y \neq y^{\prime},(x, y) \in V,\left(x, y^{\prime}\right) \in V\right\}}
$$

therefore has dimension at most 1 as soon as $2 c \geqslant \operatorname{dim}(X \times Y \times Y)-1$, or equivalently $2 \operatorname{dim}(V) \leqslant$ $\operatorname{dim}(X)+1$. When this holds, the projection $V \rightarrow X$ is finite and the lemma is proved.

Using his construction, Bogomolov exhibits smooth projective varieties with ample cotangent bundle that are simply connected. More generally, his ideas give the following result.

Proposition 26. Given any smooth projective variety $X$, there exists a smooth projective surface with ample cotangent bundle and same fundamental group as $X$. 


\section{O. DEBARRE}

Proof. By the Lefschetz hyperplane theorem, a sufficiently ample three-dimensional linear section $Y$ of $X \times \mathbb{P}^{4}$ has same fundamental group as $X$ and ample canonical bundle. A smooth hyperplane section $S$ of $Y$ with class $a h$ satisfies

$$
c_{1}^{2}(S)-c_{2}(S)=a^{2} h^{2} \cdot c_{1}(Y)+a h \cdot\left(c_{1}^{2}(Y)-c_{2}(Y)\right) .
$$

This is positive for $a \gg 0$, hence the cotangent bundle of $S$ is big by a famous trick of Bogomolov [Bog78]. Moreover, $S$ and $X$ have isomorphic fundamental groups. Starting from a simply connected $X_{0}$, we similarly obtain a simply connected surface $S_{0}$ with big cotangent bundle. Taking in Bogomolov's construction $X_{1}=\cdots=X_{5}=S_{0}$, we produce a smooth simply connected projective surface $S_{1}$ with ample cotangent bundle.

Taking in Bogomolov's construction $X_{1}=S$ and $X_{2}=\cdots=X_{5}=S_{1}$, we produce a smooth projective surface with ample cotangent bundle and same fundamental group as $X$.

\section{ACKnowledgements}

It is a pleasure to acknowledge Zak's help with the proof of Lemma 12. I am also grateful to Bogomolov for allowing me to reproduce his construction (see $\S 4$ ).

\section{REFERENCES}

BS97 Th. Bauer and T. Szemberg, Higher order embeddings of abelian varieties, Math. Z. 224 (1997), 449-455.

BD86 A. Beauville and O. Debarre, Une relation entre deux approches du problème de Schottky, Invent. Math. 86 (1986), 195-207.

BG71 S. Bloch and D. Gieseker, The positivity of the Chern classes of an ample vector bundle, Invent. Math. 12 (1971), 112-117.

Bog78 F. Bogomolov, Holomorphic symmetric tensors on projective surfaces, Uspekhi Mat. Nauk 33 (1978), 171-172, in Russian. (English transl. Russian Math. Surveys 33 (1978), 179-180.)

Bog79 F. Bogomolov, Holomorphic tensors and vector bundles on projective varieties, Izv. Akad. Nauk SSSR Ser. Mat. 42 (1978), 1227-1287, 1439, in Russian. (English transl. Math. USSR Izv. 13 (1979), 499-555.)

Deb95 O. Debarre, Fulton-Hansen and Barth-Lefschetz theorems for subvarieties of abelian varieties, J. reine angew. Math. 467 (1995), 187-197.

Deb99 O. Debarre, Tores et variétés abéliennes complexes, Cours Spécialisés, vol. 6 (Société Mathématique de France, Paris, 1999).

Dem97 J.-P. Demailly, Algebraic criteria for Kobayashi hyperbolic projective varieties and jet differentials, Algebraic geometry, Santa Cruz, 1995, Proceedings of the Symposia in Pure Mathematics, vol. 62 (American Mathematical Society, Providence, RI, 1997), 285-360.

Laz04 R. Lazarsfeld, Positivity in algebraic geometry II, Ergebnisse der Mathematik und ihrer Grenzgebiete, vol. 49 (Springer, Berlin, 2004).

Lep75 J. Le Potier, Annulation de la cohomologie à valeurs dans un fibré vectoriel holomorphe positif de rang quelconque, Math. Ann. 218 (1975), 35-53.

Mor95 A. Moriwaki, Remarks on rational points of varieties whose cotangent bundles are generated by global sections, Math. Res. Lett. 2 (1995), 113-118.

NS82 J. Noguchi and T. Sunada, Finiteness of the family of rational and meromorphic mappings into algebraic varieties, Amer. J. Math. 104 (1982), 887-900.

Sch92 M. Schneider, Symmetric differential forms as embedding obstructions and vanishing theorems, J. Algebraic Geom. 1 (1992), 175-181.

Som78 A. Sommese, Submanifolds of abelian varieties, Math. Ann. 233 (1978), 229-256. 


\section{VARIETIES WITH AMPLE COTANGENT BUNDLE}

Som79 A. Sommese, Complex subspaces of homogeneous complex manifolds. I. Transplanting theorems, Duke Math. J. 46 (1979), 527-548.

Won84 B. Wong, A class of compact complex manifolds with negative tangent bundles, in Complex analysis of several variables, Madison, WI, 1982, Proceedings of Symposia in Pure Mathematics, vol. 41 (American Mathematical Society, Providence, RI, 1984), 217-223.

Olivier Debarre debarre@math.u-strasbg.fr

Institut de Recherche Mathématique Avancée, Université Louis Pasteur, 7 rue René Descartes, F-67084 Strasbourg cedex, France 